\title{
Analysis and Countermeasures of 101 Inpatients with Falls in Internal Medicine Department
}

\author{
Xueyan Liu ${ }^{\dagger}$, Jian Yu ${ }^{\dagger}$, Shenling Hu*, Jinglan Luo*, Qingling Chen, Jiewei Huang, Yan Liu, \\ Meili Tang
}

Department of Endocrinology, The First Affiliated Hospital of Jinan University, Guangzhou, China

\section{Email address:}

10181541@qq.com (Xueyan Liu),yu38896@126.ccom (Jian Yu), 125811770@qq.com (Shenling Hu), tlj1@jnu.edu.cn (Jinglan Luo), 408783096@qq.com (Qingling Chen),dampn@126.com (Jiewei huang), 904258141@qq.com (Yan liu),527720788@qq.com (Meili Tang)

${ }^{*}$ Corresponding author

$\dagger$ Xueyan Liu and Jian Yu are co-first authors.

\section{To cite this article:}

Xueyan Liu, Jian Yu, Shenling Hu, Jinglan Luo, Qingling Chen, Jiewei Huang, Yan Liu, Meili Tang. Analysis and Countermeasures of 101 Inpatients with Falls in Internal Medicine Department. American Journal of Internal Medicine. Vol. 9, No. 2, 2021, pp. 96-101. doi: 10.11648/j.ajim.20210902.18

Received: March 5, 2021; Accepted: March 22, 2021; Published: March 30, 2021

\begin{abstract}
Falling of inpatients can cause serious injuries to patients, family members and caregivers. It damages patients' physical and mental health, increases the remedy cost of medical institution, and affects the quality of nursing service in hospital. The aim of this study is to analyze the current situation of falls of inpatients in internal medicine department, so as to provide reference information for improving patient safety management and hospital quality control. With the help of the method of retrospective investigation, the number of inpatients with falls in the First Affiliated Hospital of Jinan University from January to December 2019 was reviewed, and the incidence, causes, time and nurse patient ratio were analyzed. There were total 101 falls of inpatients in internal medicine department in year 2019, accounting for 58\% of the fall patients in the hospital, the fall rate of internal medicine department inpatients was $0.5 \%$. The injury rate of grade1 and above was $54.45 \%$. The incidence of falls has three epidemiological characteristics, mainly for the elderly over 60 years old, accounting for $71.3 \%$, the most commonplace to fall is the toilet, the high incidence period is 22:01-07:00. Drugs are one of the main factors leading to falls, with sedative-hypnoticdrugs (benzodiazepines) being the most common, followed by antidiabetic drugs, antihypertensive drugs and diuretics. Patients at high risk of falling, despite the presence of caregivers is $69.3 \%$, had a higher incidence of falls. A nurse on duty attended to 10 to 50 patients in the falling event. From the above outcome, it is illustrated that the incidence of fall injury in medical inpatients is higher. The main population is the elderly and patients taking sedatives, sleeping pills or hypoglycemic drugs Nurses need to keep an eye out for patients who take medications that can easily lead to falls. Hospital management departments should strengthen the training of nursing staff and new nurses, establish safety culture and improve nurses' consciousness of fall prevention. Head nurses should take flexible shift system to effectively reduce the incidence of falls.
\end{abstract}

Keywords: Fall, Inpatients, Internal Medicine Department

\section{Introduction}

Fall is one of the common safety events for hospitalized patients, one of the adverse nursing events, and one of the top ten safety goals in China in 2019 [1]. The incidence of falls in NDNQI (national database of nursing quality indicators) database was 3.56/1000 hospital days [2]. The incidence of serious falls in 24 geriatric wards of 12 hospitals in Australia was 9.20/1000 hospital days [3]. According to China's data in
2017, the incidence of falls in hospitalized patients was $0.054 \%$ (the number of falls in inpatients in 2019/ the actual number of bed occupied days of inpatients in $2019 \times 1000 \%$ ), and the number of falls in 490 Grade-A hospitals from 2014 to 2017 increased by $57.55 \%$ in four years. Not only would falls cause certain physical and psychological injuries to patients, increase the length of stay and expenses of patients, but also affect patients' satisfaction with medical staff [5], Therefore, more and more attention has been paid to fall prevention nursing.

Falling is defined as an unexpected event in which a 
patient falls to the ground, floor, or some lower plane [6]. For older people, a fall increases the likelihood that another fall will follow. Falls are the leading cause of disability in more than 17 million people and illness in adults over 65 years of age [7]. Falls are the most important common cause of death-associated injuries in over 75 years old [8]. Falls are costly and one of the most expensive medical conditions to treat, costing more than $\$ 50$ billion in 2015 alone [9]. Falls are more common with age, recovery time is longer, and treatment costs are higher, probably due to prolonged hospitalization [10, 11]. Furthermore, there is evidence that falls in hospitalized patients can cause minor injuries such as abrasions, lacerations, and sprains, while fractures, head and internal injuries can occur in severe cases, including hip fractures, which can reduce mobility, prolonged hospitalization and even death are the most common causes of concern [12-14]. In addition, those who experienced repeated falls are more likely to be depressed. Falls have been reported to be highly associated with depression [15].

Most of the patients in internal medicine are elderly patients with high risk of fall, and the injury caused by a fall is relatively serious, so health care providers should pay attention to the elderly patients. Fall Prevention and awareness should be assessed to reduce and possibly reduce falls at all ages. The purpose of this study was to analyze the characteristics of people who had fallen in internal medicine and to prepare for the corresponding preventive measures.

\section{Research Method}

\subsection{Data Sources}

Methods: a retrospective study was conducted on 101 patients of internal medicine department with falls in the First Affiliated Hospital of Jinan University from January to December 2019. There were 50 males and 51 females with an average age of $65.08 \pm 1.77$ years. There were 19 cases in neurology department, 15 cases in nephrology department, 13 cases in endocrinology department, 13 cases in hematology department, 12 cases in cardiology department, 10 cases in respiratory department, 10 cases in psychiatric department, 4 cases in digestive department, 1 case in traditional Chinese medicine department, 3 cases in oncology department and 1 case in comprehensive department.

\subsection{Research Indicators}

Fall is defined as an unexpected fall to the ground or lower than the initial position in any place in a medical institution, with or without trauma. Fall injury is defined by international standards: Grade 1 (mild): the degree of injury that does not need or needs only light treatment and observation, such as abrasions, contusions, small tears of the skin that do not need to be sutured, etc. Grade 2 (moderate): degree of injury such as sprain, large or deep tear, skin tear or small contusion; which is requiring medical or nursing treatment and observation such as ice compress, bandage, suture or splint. Grade 3 (severe): the degree of injury such as fracture, loss of consciousness, mental or physical state change requiring medical treatment and consultation. Grade 4 (death): the patient died of persistent injury caused by a fall.

The fall incidence rate of inpatients=the number of falls in inpatients in a certain time/ the actual number of bed occupied days of inpatients in the same time $\times 1000 \%$.

\subsection{Statistical Methods}

SPSS 24.0 software was used to analyze the data. Count data are expressed by frequency and rate $(\%)$, and measurement data are expressed by mean \pm standard deviation $\left(\overline{\mathrm{X}}_{\mathrm{x}}\right)$.

\section{Result}

\subsection{Basic Information of Patients}

Table 1. Basic information of 101 patients.

\begin{tabular}{|c|c|}
\hline project & constituent ratio $\mathbf{N}(\%)$ \\
\hline male & $50(49.5)$ \\
\hline female & $51(50.5)$ \\
\hline \multicolumn{2}{|l|}{ Age } \\
\hline$<50$ & $16(15.8)$ \\
\hline $50 \sim 59$ & $13(12.9)$ \\
\hline $60 \sim 69$ & $21(20.8)$ \\
\hline $70 \sim 79$ & $26(25.7)$ \\
\hline$\geq 80$ & $25(24.8)$ \\
\hline \multicolumn{2}{|l|}{ diagnosis } \\
\hline diabetes & $12(11.9)$ \\
\hline Cerebrovascular complications & $14(13.9)$ \\
\hline Mental illness & $11(10.9)$ \\
\hline Renal complications & $12(11.9)$ \\
\hline Retinopathy & $1(1.0)$ \\
\hline tumour & $6(5.9)$ \\
\hline Pulmonary diseases & $9(8.9)$ \\
\hline Digestive system diseases & $4(4.0)$ \\
\hline Cardiovascular diseases & $12(11.9)$ \\
\hline Hematopathy & $11(10.9)$ \\
\hline other & $9(8.9)$ \\
\hline \multicolumn{2}{|l|}{ mentality } \\
\hline Conscious & $97(96.0)$ \\
\hline Disorientation & $1(1.0)$ \\
\hline Non directional force & $1(1.0)$ \\
\hline Fidgety & $2(2.0)$ \\
\hline \multicolumn{2}{|l|}{ Activity ability } \\
\hline Auxiliary support & $40(39.6)$ \\
\hline free activity & $46(45.5)$ \\
\hline Bed rest & $3(3.0)$ \\
\hline Slow to move & $12(11.9)$ \\
\hline \multicolumn{2}{|l|}{ Disability } \\
\hline Yes & $5(5.0)$ \\
\hline No & $96(95.0)$ \\
\hline \multicolumn{2}{|l|}{ Oral medication of patients } \\
\hline Hypnotic sedative & $20(19.8)$ \\
\hline Hypoglycemic agents & $10(9.9)$ \\
\hline Hypotensives & $8(7.9)$ \\
\hline Diuretic & $8(7.9)$ \\
\hline \multicolumn{2}{|l|}{ Is it a high risk group } \\
\hline Yes & $80(79.2)$ \\
\hline No & $21(20.8)$ \\
\hline
\end{tabular}

In terms of age characteristics, 72 cases $(71.30 \%)$ were over 60 years old, 47 cases ( $46.53 \%$ ) were between 60 and 79 years old, and 25 cases $(24.8 \%)$ were over 80 years old. The patients diagnosed with cerebrovascular complications accounted for the largest proportion, $13.9 \%$. Patients taking hypnotic 
sedatives accounted for the largest proportion, $19.8 \%$. Detailed information can be found in Table 1.

\subsection{Basic Information of Patients with Falls}

The toilet was the place where the most falls occurred (48 cases, 47.5\%). Most falls occur at night, from 22:01 to 07:00. A nurse usually take care of 10 or more patients at day and more than 20 patients at night. Detailed information can be found in Table 2 .

Table 2. Specific conditions of patients with falls.

\begin{tabular}{|c|c|}
\hline project & constituent ratio $\mathrm{N}(\%)$ \\
\hline \multicolumn{2}{|l|}{ Where the fall occurred } \\
\hline Toilet & $48(47.5)$ \\
\hline beside the hospital bed & $33(32.7)$ \\
\hline Ward corridor & $11(10.9)$ \\
\hline Out of ward & $6(5.9)$ \\
\hline Ward balcony & $3(3.0)$ \\
\hline \multicolumn{2}{|l|}{ Time of fall } \\
\hline 07:01-09:00 & $10(9.9)$ \\
\hline 09:01-12:00 & $8(7.9)$ \\
\hline $12: 01-15: 00$ & $11(10.9)$ \\
\hline $15: 01-18: 00$ & $16(15.8)$ \\
\hline $18: 01-22: 00$ & $10(9.9)$ \\
\hline 22:01-07:00 & $46(45.5)$ \\
\hline \multicolumn{2}{|l|}{ Fall season } \\
\hline first quarter & $24(23.8)$ \\
\hline Second quarter & $31(30.7)$ \\
\hline Third quarter & $26(25.7)$ \\
\hline Fourth quarter & $20(19.8)$ \\
\hline \multicolumn{2}{|l|}{ The process of falling } \\
\hline Defecate or urinate & $56(55.4)$ \\
\hline Up and down the bed & $31(30.7)$ \\
\hline bath & $6(5.9)$ \\
\hline walking & $4(4.0)$ \\
\hline Wheelchair transfer & $2(2.0)$ \\
\hline Sit and stand & $2(2.0)$ \\
\hline \multicolumn{2}{|l|}{ Grading of fall injury } \\
\hline No harm & $46(45.5)$ \\
\hline 1 & $45(44.6)$ \\
\hline 2 & $6(5.9)$ \\
\hline 3 & $4(4.0)$ \\
\hline \multicolumn{2}{|l|}{ Accompany } \\
\hline Yes & $70(69.3)$ \\
\hline No & $31(30.7)$ \\
\hline \multicolumn{2}{|c|}{ Number of patients attended by nurses } \\
\hline$<10$ & $10(9.9)$ \\
\hline $10-20$ & $21(20.7)$ \\
\hline $21-30$ & $12(11.9)$ \\
\hline$>30$ & $30(29.7)$ \\
\hline Missing & $28(27.7)$ \\
\hline \multicolumn{2}{|c|}{ Working years of responsible nurses } \\
\hline$<5$ & $55(54.5)$ \\
\hline $6 \sim 10$ & $35(34.7)$ \\
\hline $11 \sim 15$ & $7(6.9)$ \\
\hline $16 \sim 20$ & $3(3.0)$ \\
\hline Missing value & $1(1.0)$ \\
\hline
\end{tabular}

\section{Discussion}

\subsection{The Incidence of Falls Is High and the Injury Rate Is High}

According to the fall data of the first affilated hospital of JiNan University in 2019, the fall rate of inpatients in internal medicine is
$0.5 \%$, accounting for $58 \%$ of the fall patients in our hospital, which is higher than that in surgery and other departments. The main reasons are as follows: firstly, compared with surgical inpatients, patients in internal medicine are older. $71.3 \%$ of the elderly patients are over 60 years old. As we get older, our balance deteriorates and we tend to fall over when we walk. At the same time, most of the elderly patients are prone to postural hypotension and fall immediately when going to the toilet. The highest incidence of falls occurred in the process of defecating or urinating, which is consistent with Jiang Xuexia's research [16]. Secondly, most of the inpatients in internal medicine have a variety of complications. Some studies have shown that the incidence of falls is positively correlated with the number of disease diagnose [17]. Lastly, the main oral drugs for medical inpatients are hypoglycemic, hypotensive, diuretic and sedative drugs, which are high risk drugs for falls. The fall injury rate of grade 1 and above in this survey was $54.45 \%$, and the fall injury grading was mainly concentrated in Grade 1 (mild injury). The degree of injury that does not need or only needs light treatment and observation, such as scratch, contusion, small skin tear that does not need suture, etc. This is close to the research results of $\mathrm{Wu}$ Xiaoqun [18], and the fall injury rate is $52.24 \%$. The incidence of falls in the national database of nursing quality indicators (NDNQI) database of the United States is 3.56/1000 hospital days, of which the injury rate is $26.1 \%$ [2]. The data in this study is higher than that in the United States, which is different from the situation reported for falls in each place. At the same time, the longer the length of hospital stay, the higher the risk of falls. The length of hospital stay of inpatients in China is longer than that in foreign countries.

\subsection{The Occurrence of Falls Has Three Distribution Characteristics}

\subsubsection{Population Distribution of Falls}

According to the data, more than one third of the elderly over 65 years old fall every year [19]. Due to their poor balance ability, the elderly are more likely to fall. Some studies have shown that the balance and movement disorders of elderly patients are independent risk factors for falls [20]. Therefore, we can increase some exercise items to improve the balance of patients, such as standing, walking and so on [21-23]. Although this study showed no statistically significant difference between men and women, some researchers believe that women are more likely to fall after menopause because their estrogen levels drop, leading to osteoporosis and metabolic bone hyperplasia [23].

\subsubsection{Location Characteristics of Falls}

According to the data of this study, the toilet was the place where the most falls occurred (48 cases, $47.5 \%$ ). This relates to the main activity they engage in, defecating, urinating or bathing. The main factors were as follows: the patient was afraid to disturb other patients in the middle of the night without turning on the light, so he accidentally tripped over the obstacle; he fell down due to weakness of both lower limbs (35 cases) or getting up too quickly; he fell down due to wet and slippery toilet floor. Therefore, we need to conduct patients from lying to standing to walk in three steps, install alarms in the toilet and 
take anti-skid measures in the ward and toilet. Try to use anti-skid tiles or guide patients to wear anti-skid shoes. Studies have shown that targeted guidance for inpatients using the American inpatient fall prevention model (ATTEND) can effectively reduce the risk of falls [24]. The attend model includes six contents: (1) assessment (A): the Morse Fall assessment scale was used to assess the risk factors of falls when the patients were admitted to hospital. If the score was more than 45 , they were rated as high risk of falls and needed intervention. (2) Treatment (T): firstly, evaluate the medication situation of patients. Secondly, carry out purposeful inspection with $5 p$ inspection method [25], that is, carry out periodic inspection on potty, pain, position, possession, pumps. Finally, carry out rehabilitation training for patients, and guide them to carry out fall prevention exercises.(3) Toilet (T): on-site guidance and demonstrations on proper toilet use should be given by nurses when patients are admitted; pagers and handrails should be installed in the toilets; family members or nurses should accompany high-risk patients when using the toilets. (4) Engagement (E): both patients and their families participate in fall prevention management. (5) Never (N): don't leave high-risk patients in a room alone. (6) Debrief (D): the nursing department adopts non punitive measures to encourage nurses to report each fall, the Department analyzes the causes of the fall and carries out rectification, and the nursing safety committee follows up, guides and supervises the implementation of rectification measures. Moreover, nurses should pay more attention to the safety of patients and their families in the mode of ATTEND [24].

\subsubsection{Time Distribution Characteristics of Fall Patients}

The results in Table 2 show that the fall time of patients is mainly from $22: 01$ to $07: 00$. There are three possible main reasons: (1) during this period of time, the nursing manpower in the ward is the weakest, and there is generally one night shift nurse, but there are as few as 20 patients and as many as 50-60 patients. In addition, the accompanying personnel is in the deep sleep condition during this time period, easy to neglect the patient. (2) Elderly male patients with benign prostatic hyperplasia, bladder volume is relatively reduced, resulting in increased frequency of going to toilet. This is the main reason for falls [26]. (3) People are in a fuzzy sleep state at night, which is easy to lead to weak photosensitive consciousness, while elderly patients are more likely to fall down at night due to the decline of perception and balance. In terms of the season, the results show that the fall rate is the highest in the second quarter. In southern China, Guangdong is in the Meiyu season in the second quarter, and air and ground are wet, so it is more prone to fall. The abnormal patients should be taken as the key objects to prevent falls at night, for high-risk fall patients, nurses should strengthen the frequency of inspection, accompanying staff should increase vigilance and sense of responsibility.

\subsection{Effect of Drugs on the Occurrence of Falls}

The risk of falls in the hospital of patients with hypertension is about 9 times higher than that of other patients due to more complications and combination of drugs [27]. Diabetic patients with lower limb sensory neuropathy and decreased muscle strength lead to decreased balance ability [28, 29], resulting in gait instability and increased fall risk. Therefore, the patients with hypertension and diabetes history should be evaluated with lower limb muscle strength and Assessment of daily living ability (ADL) when they are admitted to hospital. The results of Table 1 show that the patients taking sedative hypnotic drugs have the highest incidence of falls (19.8\%), and the hypnotic drugs taken are mainly benzodiazepines, followed by hypoglycemic drugs, antihypertensive drugs and diuretics. Japanese researcher Kyoko [30] analyzed the fall events of 3683 inpatients in a Japanese hospital. The data showed that the highest incidence of falls was $2.5 \%$ when taking hypnotic drugs, followed by antiepileptic drugs, opioids, Alzheimer's disease, hypoglycemic drugs, antihypertensive drugs and antiarrhythmic drugs. Other studies have also shown that taking benzodiazepines can increase the risk of falls [31, 32], but appropriate selection of hypnotics and assessment of related risks can effectively reduce the risk of falls [30]. Benzodiazepines are benzodiazepines (BZ) receptors that selectively excite the central nervous system, while non -benzodiazepines are selective bz1 ( $\left.\begin{array}{ll}\omega & 1\end{array}\right)$ receptor agonists. Both of them can produce sedative and hypnotic effects. However, benzodiazepines have muscle relaxant effect and relatively high risk of falls. Non benzodiazepines do not have muscle relaxant effect, so it is not easy to make problem of the balance for patients. It is considered to be a relatively safe sedative hypnotic [33, 34]. The adverse reactions of the above antihypertensive drugs, hypoglycemic drugs and diuretics are the risk factors for patients to fall [35].

\subsection{Influence of Nursing Workers on Fall of Patients}

In this survey, $69.3 \%$ of the patients with falls were accompanied by nursing workers, but the incidence of falls was still very high, mainly due to the lack of ability of nursing workers to assess the risk of falls, poor sense of responsibility and lack of supervision and assessment of nursing workers [36]. The main purpose of nursing workers is to take care of patients' daily life, which has become an indispensable labor supplement in nursing work. However, due to the inadequate training and different standards, as well as the differences in education level and individual quality of nursing workers, the service content and service quality provided by nursing workers for patients are uneven [37]. In the United States, Britain, Japan and other countries, nursing workers will be included in the hierarchical management system of hospital nursing staff, and their job responsibilities will be clarified [38]. Although China has also issued corresponding rules and regulations to standardize the management of nursing workers, its binding force is still weak, the implementation is insufficient, and the satisfaction of patients and their families to nursing workers needs to be improved [37]. How to carry out systematic and standardized training for nursing workers, reasonably play its role, and meet the higher needs of patients, family members and the hospital is a problem that hospital 
managers need to explore and think about [36]. Studies have shown that hierarchical training mode can improve the clinical care ability of nursing workers and the satisfaction of patients and their families $[39,40]$.

\subsection{Influence of Nurses on Patients' Fall}

The results in Table 2 show that $54.5 \%$ of the patients' responsible nurses are less than 5 years old, and the nurses with short working years lack the ability to comprehensively evaluate the patients. The researcher Jacqueline [41] conducted a survey on the knowledge of fall prevention among 147 nursing staff, and found that only 39 (26.5\%) knew that their nursing patients were at risk of falling. Therefore, nursing managers should strengthen the training for nursing staff, especially for new nurses with less qualifications, strengthen their awareness of fall prevention, and improve their ability to assess patients to reduce the occurrence of adverse events. In the proportion of nurses to patients, a nurse usually take care of 10 or more patients at day and more than 20 patients at night. Through reviewing 335 nursing adverse events in 2008 in China, Yang Xin [42] found that the more patients needed to be cared, the higher the incidence of adverse events. Therefore, the lack of nursing manpower is also one of the main factors leading to the occurrence of fall adverse events. Hospital administration should increase the support of nursing staff, increase nurses, so that more nursing staff can directly take care of patients. Head nurses should adopt flexible scheduling system [43], especially should pay attention to rest days, night and other periods, should reasonably arrange nursing human resources according to the needs of patients, reduce the occurrence of falls during hospitalization. At the same time, the number of caregivers can be increased at night, but how to adjust and assess the working mode of caregivers is also a problem that managers need to solve.

\section{Conclusion}

This study is mainly for internal medicine inpatients fall situation analysis, fall injury rate is high, patients fall during hospitalization lead to injury will prolong the length of stay. The risk factors are patients' poor muscle, drugs, poor accompanying and nursing human resources and other factors. Therefore, nursing staff should evaluate each hospitalization patients carefully and take preventive measures, especially for those who use benzodiazepines, hypoglycemic drugs, antihypertensive drugs, diuretics and other drugs. Hospital administrator should improve the management system of nursing workers with a training system, increase the support of nursing staff, strengthen the training of new nurses, establish an organizational safety culture to prevent the occurrence of adverse events of falls. At the same time, head nurse should adopt flexible shift scheduling according to the needs of patients, which can effectively reduce the occurrence of adverse events of falls.

\section{Suggestions for Future Studies}

It is suggested that a multidisciplinary study should be conducted on patients in the department of internal medicine who are taking benzodiazepines, sedatives, hypoglycemic drugs and antihypertensive drugs, with emphasis on their activities of getting up, adding safety fall prevention devices in toilets, and reducing the incidence of falls in toilets.

\section{References}

[1] China Hospital Association. Patient safety objectives of Chinese Hospital Association (2019). China Health, 2019, 12: 57-58.

[2] Bouldin E L D, Andresen E M, Dunton N E, et al. Falls Among Adult Patients Hospitalized in the United States: Prevalence and Trends. Jouanal of Patien Safety, 2012, 9 (1): 13.

[3] The Joanna Briggs Institute, Li Ying, Hu Yan. Fall prevention of hospitalized elderly patients. Chinese Journal of nursing, 2013, 48 (6): 574-576.

[4] Wu Zhijun, Shang Wenhan, Jian Weiyan, et al. Analysis of the incidence of falls in hospitalized patients in China: A survey of 490 tertiary hospitals. Health quality management in China, 2019, 3 (26): 14-17.

[5] Kim EA, Mordiffi SZ, Bee WH, et al. Evaluation of three fall-risk assessment tools in an acute care setting. J Adv Nurs. 2007; 60 (4): 427-35.

[6] K. Kimiafar, M. Farrokhi, F. Manouchehri Monazah et al., Fall-related hospitalization of patients in Iran, Chinese Journal of Traumatology, https://doi.org/ 10.1016/j.cjtee.2021.01.001.

[7] Magruder KM, Kassam-Adams N, Thoresen S, Olff M. Prevention and public health approaches to trauma and traumatic stress: a rationale and a call to action. Eur J Psychotraumatol. 2016; 7: 29715.

[8] Shrestha R, Shrestha S, Kayastha S, Parajuli N, Dhoju D, Shrestha D. A comparative study on epidemiology, spectrum and outcome analysis of physical trauma cases presenting to emergency department of Dhulikhel Hospital, Kathmandu University Hospital and its outreach centers in rural area. Kathmandu Univ Med J. 2013; 11: e6.

[9] Centers for Disease Control and Prevention [CDC]. (2016). About CDC's STEADI (stopping elderly accidents, deaths, \& injuries) initiative. Retrieved from https://www.cdc.gov/steadi/about.html.

[10] Bergen, G., Burns, E., \& Stevens, M. Falls and fall injuries among adults aged $\geq 65$ years - United States, 2014. Morbidity and Mortality Weekly Report, 2016, 65 (37): 5.

[11] Frith, K., Hunter, A., Coffey, S., \& Khan, Z. A longitudinal fall prevention study for older adults. The Journal for Nurse Practitioners, 2019, 15 (4): 295-300.

[12] Kannus P, Sievänen H, Palvanen M. Prevention of falls and consequent injuries in elderly people [J]. Lancet, 2005, 366 (9550): 1885-1893.

[13] Li, Weal., The Association Between Painand Fall Among Middle-Aged and Older Chinese, Pain Management Nursing, https://doi.org/10.1016/j.pmn.2020.10.004. 
[14] Hubert Blain, Lucie Gamon, Bérangère Aliaga, et al. Self- rep orted fatigue: A significant risk factor for falling in older women and men. Experimental Gerontology, 2021, 143: 1-6.

[15] Jae-Hyun Kim. Experiences of falling and depression: Results from the Korean Longitudinal Study of Ageing. Journal of Affective Disorders, 2021, 281: 174-182.

[16] Jiang Xuexia, Lou min, Xiao Na, et al. Analysis of three distribution characteristics of inpatient falls. Journal of nursing education. 2019, 19 (34): 1777-1779.

[17] Shen Mingyan, Wang Huafen, Feng Xiuqin, et al. Analysis of data mining and fall prevention countermeasures for inpatients with falls. Chinese Journal of nursing, 2017, 52 (9): 1087-1091.

[18] Wu Xiaoqun, Huang Feng. Effectiveness analysis of fall risk management for inpatients. Journal of nursing, 2018, 33 (5): 45-47.

[19] Todd C, Skelton D: What are the main risk factors for falls among older people and what are the most effective interventions to prevent these falls. Copenhagen: World Health Organisation; 2004.

[20] Deandrea S, Lucenteforte E, Bravi F, et al. Risk factors for falls in community-dwelling older people: a systematic review and meta-analysis. Epidemiology. 2010; 21 (5): 658-668.

[21] Elissa, Burton, Vinicius, et al. Effectiveness of exercise programs to reduce falls in older people with dementia living in the community: a systematic review and meta-analysis. Clinical Interventions in Aging 2015: 10 421-434.

[22] Suttanon P, Hill KD, Said CM, et al. Feasibility, safety and preliminary evidence of the effectiveness of a home-based exercise programme for older people with Alzheimer's disease: a pilot randomized controlledtrial. ClinRehabil. 2013; 27 (5): 427-438.

[23] Robertson M, Campbell AJ, Gardner M, Devlin N. Preventing injuries in older people by preventing falls: a meta-analysis of individual-level data. J Am Geriatr Soc. 2002; 50 (5): 905-911.

[24] Xu Yijun, Li Zhenyun, Fu Wenquan, et al. Application of fall prevention model for inpatients in the United States in elderly patients with type 2 diabetes mellitus. Nursing research, 2020, 34 (6): 1102-1105.

[25] Bonuel N, Manjos A, Lockett L et al. Best practice fall prevention strategies [J]. Critical Care Nursing Quarterly, 2011, 34 (2): 154-158.

[26] Wang Xiaoai, Hou Huiru, Yang Li, et al. Analysis and nursing countermeasures of falls in 75 elderly patients in hospital. Journal of nursing management. 2011, 11 (4): 290.

[27] Milos V, Bondesson A, Magnusson M, et al. Fall risk increasing drugs and falls: a cross sectional study among elderly patients in primary care. BMC Geriatrics, 2014, 14 (40): 17.

[28] Landi F, Liperoti R, Russo A, et al. Sarcopenia as a risk factor for falls in elderly individuals: results from the ilSIRENTE study [J]. Clin Nutr, 2012, 31 (5): 652-658.

[29] Fang Qunhui, Zhou Weifeng, Tang Songtao, et al. Analysis of related risk factors of balance ability in elderly patients with type 2 diabetes mellitus. Chinese Journal of diabetes, 2014, 6 (5): 308-311.

[30] Obayashi K, Araki T, Nakamura K, et al. Risk of Falling and Hypnotic Drugs: Retrospective Study of Inpatients. Drugs R D. 2013, 13 (2): 159-64.

[31] Yi Yanzhi, Shannon H Houser, Shi Lanping, et al. Predictive analysis of fall risk factors of inpatients. Nursing research, 2019, 33 (9): 1593-1596.

[32] Chang CM, Chen MJ, Tsai CY, et al. Medical conditions and medications as risk factors of falls in the inpatient older people: a case-control study. Int J Geriatr Psychiatry. 2011, 26 (6): $602-7$

[33] Shirakawa K. Pharmacological profile and clinical effect of zolpidem (Myslee tablets), a hypnotic agent. Folia Pharmacol Jpn. 2002, 119: 111-8.

[34] Hanson SM, Morlock EV, Satyshur KA, Czajkowski C. Structural requirements for eszopiclone and zolpidem binding to the gammaaminobutyric acid type-A (GABA (A)) receptor are different. J Med Chem. 2008, 51: 7243-52.

[35] Liu Huagang, Huang Renbin, Wu Chuang, Li Mei. Clinical practical Drug Handbook [M]. Beijing People's Health Publishing House, 2014.

[36] Fu Xia, Lu Shaoping. Research Progress on standardized management of full-time caregivers in hospitals in China. Nursing research, 2006, 20 (24): 2163-2165.

[37] Pengdengbing. Investigation on the satisfaction of elderly inpatients to nursing work. Electronic Journal of practical clinical nursing, 2017, 2 (3): 141, 143.

[38] Yuan Qun, Yi Xia, Zhang Yinhua, et al. Research status and progress of work stress of nursing staff for the aged. Nursing management in China, 2015, 15 (1): 112-115.

[39] Roitenberg N. Ethno-national boundaries in the construction of "dirty-work" occupational identity: the case of nursing care workers in diversified workplaces [J]. International Journal Of Inercultural Relations, 2020, 5 (76): 1-12.

[40] Zhao Wenjing, Wang Ying, Han Jie, et al. Study on the effect of hierarchical training for nursing workers based on the needs of patients. Chinese Journal of Modern Nursing, 2020, 26 (32): 4533-4536.

[41] Francis-Coad J, Hang JA, Etherton-Beer C, et L. Evaluation of care staff knowledge, confidence, motivation and opportunity for preventing falls in residential aged care settings: A cross-sectional survey. Int J Older People Nurs. 2019, 14 (2): e12224.

[42] Yang Shen, Wang Xiang, Shao Wenli, et al. Analysis and Countermeasures of 335 nursing adverse events. Chinese Journal of nursing. 2010, 45 (2): 130-132.

[43] Wang Lijin, Huang Xuemei, Li Yan, et al. Investigation and analysis of nursing workload and manpower allocation in respiratory department. Nursing management in China, 2009, 9 (5): 29-31. 